\title{
Structural Topology Optimization based on Parametric Level Set Method under the Environment of ANSYS Secondary Development
}

\author{
Xin Gui, Mi Xiao a, ${ }^{*}$, Yan Zhang, Liang Gao, Yuancan Liao \\ State Key Laboratory of Digital Manufacturing Equipment and Technology, Huazhong University of \\ Science and Technology, Wuhan 430074, China \\ axiaomi@hust.edu.cn
}

Keywords: Topology optimization, parametric level set method, ANSYS secondary development.

\begin{abstract}
As the parametric level set method (PLSM) can provide the clear structural boundary description and the ANSYS software has a strong capability of finite element analysis, structural topology optimization based on PLSM under the environment of ANSYS secondary development is conducted in this paper. The finite element analysis programs and structural topology optimization based on PLSM are written by APDL and UPFs, respectively. This approach based on ANSYS secondary development can expand the practical applications of structural topology optimization. Typical 2D and 3D examples shown in this paper are used to verify the feasibility and applicability of the adopted method, which can also provide an important guidance to achieve structural topology optimization under the environment of ANSYS secondary development.
\end{abstract}

\section{Introduction}

As the most challenging research direction in the field of structural optimization, topology optimization is becoming an indispensable optimization tool in the engineering area [1, 2], such as aerospace, automobile industry, biological engineering, material engineering and so on. Compared with the traditional structural optimization methods, topology optimization was establishing an overall framework of conceptual design without prior knowledge of an optimal shape and size design [3]. The topology optimization has experienced considerable progress during the past three decades, and several well-known methods such as homogenization method [3], Solid Isotropic Material with Penalization (SIMP) [4, 5], Evolutionary Structural Optimization (ESO) [6] and level set method (LSM) [7, 8] have been proposed.

The SIMP-based topology optimization method has experienced considerable popularity due to its conceptual simplicity and numerical easiness, and it has been favored by some commercial software vendors such as ANSYS and HyperWorks. However, this method is usually undergone numerical instabilities during optimization process, and the resulting boundary of the optimized design is usually identified with zigzag features and ambiguities boundary, which means that additional smoothing scheme should be included to reconstruct a geometrical model in order to facilitate the fabrication of the final design [9].

To deal with the zigzag boundary and ambiguities of intermediate element densities of the SIMP-based topology optimization method, Sethian and Wiegmann [7] firstly introduced the level set method (LSM) into structural topology optimization by the Eulerian representation to make the topology and shape merging and breaking naturally. The LSM can provide unique benefits in shape and topology optimization, for example smooth boundary and distinct interface, shape fidelity and topological flexibility. However, the conventional LSM is often hampered by several unfavorable numerical features in topology optimization process, such as velocity extension, re-initialization and Courant-Friedrichs-Lewy (CFL) condition [10]. To avoid the above mentioned numerical problems in the conventional LSM, the efficient PLSM [11,12] are proposed as an alternative for the topological shape optimization of structures. In this method of PLSM, the compactly supported radial basis function (CSRBF) [13, 14] was utilized to achieve the interpolation of the implicit level set function, and then the design boundary was advanced by updating a set of the expansion coefficients. 
The PLSM has shown its ability as a powerful topological shape optimization method [15, 16], which can remain favorable aspects while avoid unfavorable numerical issues of the conventional LSM.

ANSYS, as one of the world's largest finite element analysis software, has been widely used in the analysis of structure, fluid, electric filed, magnetic field and sound field. ANSYS not only has strong finite element analysis capability, but also has the convenient secondary development tools that provide users with the possibility of designing new unit types, material propertied, optimization algorithms and so on.

Based on the above description, due to the advantages of PLSM as well as the strong finite element analysis capability of ANSYS, structural topology optimization based on PLSM under the environment of ANSYS secondary development is produced in this paper. Because of the convenient secondary development tools of ANSYS, we design finite analysis programs and topology optimization based on PLSM by APDL and UPFs, respectively. Several 2D and 3D examples are used to test the produced method. It is illustrated that the method is feasible and correct, which can extend the practical applications of structural topology optimization. Moreover, this work can provide an important guidance to achieve structural topology optimization under the environment of ANSYS secondary development.

\section{Representation of parametric level set method}

The level set method was firstly introduced into structural optimization by Sethian and Wiegmann [7] to enable the dynamic process of shape boundary. The pseudo-time $t$ is introduced into the Lipschitz continuous level set function $\Phi(\mathbf{x})$, which is defined over a reference domain $D \subset R^{d}(d=2$ or 3$)$ including all admissible shapes $\Omega(\Omega \subset D)$. For $d=2$, the scheme can be defined as follows:

$$
\begin{cases}\Phi(\mathbf{x}, t)<0 \Leftrightarrow \forall \mathbf{x} \in D \backslash \Omega & \text { (void region), } \\ \Phi(\mathbf{x}, t)=0 \Leftrightarrow \forall \mathbf{x} \in \partial \Omega \cap D & \text { (design boundary), } \\ \Phi(\mathbf{x}, t)>0 \Leftrightarrow \forall \mathbf{x} \in \Omega \backslash \partial \Omega & \text { (solid region). }\end{cases}
$$

where $\Phi$ can be described random shape freely, and $\mathbf{x}$ represent the knot coordinates in higher dimensional space.

Taking pseudo time $t$ into the dynamic process of shape deformations and differencing $\{\Phi(\mathbf{x}, t)=0\}$ on both sides, one can obtain the following Hamilton-Jacobi equation [17].

$$
\frac{\partial \Phi(\mathbf{x}, t)}{\partial t}-v_{n}|\nabla \Phi|=0, \quad \Phi(\mathbf{x}, 0)=\Phi_{0}(\mathbf{x})
$$

The normal velocity is expressed as follows:

$$
\mathbf{v}_{n}=\mathbf{v} \cdot \frac{\nabla \Phi}{|\nabla \Phi|}=\frac{d \mathbf{x}}{d t} \frac{\nabla \Phi}{\sqrt{\nabla \Phi \cdot \nabla \Phi}}
$$

Hence, moving the boundary $\Gamma=\{\mathbf{x} \mid \Phi(\mathbf{x})=0\}$ along the norm direction $\mathbf{n}=\nabla \Phi /|\nabla \Phi|$ is equivalent to transporting $\Phi$ by solving the Hamilton-Jacobi partial differential equation (2). The representation scheme is illustrated in Fig. 1(a) and 1(b) for the case of $d=2$.

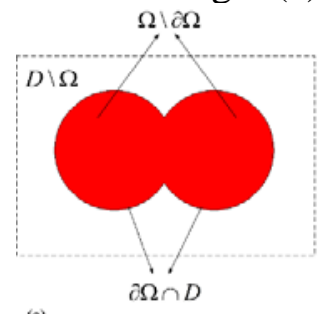

(a)

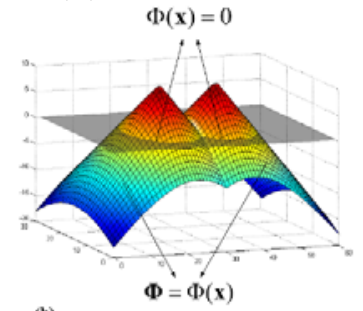

(b)

Fig. 1 Design domain and the level set function: (a) solid parts and its embedding domain D; (b) level set function and its zero level set 
However, a general analytical function for the scalar level set function is usually unknown [18]. Thus, the discretization through a distance transform is necessary for level set processing. As aforementioned, numerical scheme, extension velocities and re-initialization algorithms must be proper settled to meet the numerical requirements for the discretization. These numerical difficulties limit the application of standard LSM to shape and topology optimization problems.

\subsection{Parametric level set method}

In order to eliminate this issue, Wang [19], Luo et al [20] proposed the parametric shape representation, which parameterize with continuous, differentiable functions based implicit RBFs model. Luo [18] introduced CSRBF into level set method, converting the level set function as a parametrical form. The CSRBFs with C2 proposed by wendland [21] can be written as follows:

$$
\varphi(r)=\max \{0,(1-r)\}^{4}(4 r+1)
$$

Here, for 2D case, the radius of support $r$ defined in a Euclidean space can be given as:

$$
r=\frac{d_{i}}{d_{m i}}=\frac{\sqrt{\left(x-x_{i}\right)^{2}+\left(y-y_{i}\right)^{2}}}{d_{m i}}
$$

where the parameter $d_{i}$ represents the distance between the current sample knots $(x, y)$ and the interpolation control knots $\left(x_{i}, y_{i}\right)$ within the supporting radius, and the parameter $d_{m i}$ indicates the supporting radius of influences from the other adjacent knots, which can be calculated by using $d_{m i}=d_{\max } \cdot C_{i}$, where $d_{\max }$ is a scaling parameter factor typically 2.0-4.0 for a static analysis, and $C_{i}$ is used to guarantee there are enough knots located in the neighborhood for the current knot [21].

Now, we turn to RBF interpolation, the basic RBF approximation to $f(x)$ is given by the expansion:

$$
\widetilde{f}(x)=\sum_{i=1}^{N} \alpha\left(x_{i}\right) \varphi\left(\left\|x-x_{i}\right\|\right)
$$

where $\|\bullet\|$ denotes the d-dimensional Euclidean norm, $x_{i}$ presents the interpolation control knots’ location, $\alpha\left(x_{i}\right)$ is the expansion coefficient, and $\varphi$ is the base function.

Then, the level set function is transformed by the CSRBF-based interpolation, so the level set function is transformed to the function that is independent with spatial variable $\mathbf{x}$ and the time variable $t$, which can be written as:

$$
\Phi(\mathbf{x}, t)=\boldsymbol{\varphi}(x) \boldsymbol{\alpha}(t)=\sum_{i=1}^{N} \varphi_{i}(x) \alpha_{i}(t)
$$

where $\boldsymbol{\varphi}(x)$ is set of the interpolation function of RBF model, $\boldsymbol{\alpha}(t)$ is the expansion coefficient related with time in the iterations.

Combine Eqs. (2) and (7), the moving level set model is rewritten as:

$$
\boldsymbol{\varphi}(x) \frac{d \boldsymbol{\alpha}(t)}{d t}-v_{n} \cdot \nabla \boldsymbol{\varphi}(x) \boldsymbol{\alpha}(t) \mid=0
$$

Then, the $\mathbf{v}_{n}$ related to the expansion coefficients is given as:

$$
v_{n}=\frac{\varphi(x)}{|\nabla \boldsymbol{\varphi}(x) \boldsymbol{\alpha}(t)|} \cdot \frac{d \boldsymbol{\alpha}(t)}{d t}
$$

Hereto, the CSBRF has been applied to convert the conventional LSM to a parametric form. Thus, the time-consuming process of handing Hamilton-Jacobi PDE is replaced by solving the ODEs. In addition, Eq. (8) can be solved by normal, simply gradient-based method, such as the steepest descent method, the moving asymptote (MMA) method and the optimality criteria (OC) method.

\subsection{Sensitivity analysis and optimality criteria method}

For the sake of simplicity but without losing generality, we discuss the famous optimization problem of minimizing the compliance for linear elastic structure. The mathematical model based on the level set method is established as: 
Find : $\quad \boldsymbol{\alpha}=\left[\alpha_{1}, \alpha_{2}, \ldots, \alpha_{N}\right]^{T}$

$\underset{(u, \Phi)}{\operatorname{Minimize}:} \quad J(u, \Phi)=\int_{\Omega} f(u, v) H(\Phi) d \Omega$,

Subject to: $G(\Phi)=\int_{\Omega} H(\Phi) d \Omega \leq V_{\max }$,

$$
\begin{aligned}
& a(u, v, \Phi)=l(u, v),\left.u\right|_{\partial \Omega}=u_{0}, \forall v \in U, \\
& \alpha_{i, \min } \leq \alpha_{i} \leq \alpha_{i, \max } .
\end{aligned}
$$

where the inequality constraint is introduced to limit the material usage, and $v_{\max }$ is the prescribed volume, $u$ is the displacement field, $v$ is the virtual displacement field belonging to the space $\mathbf{U}$ spanned by the kinematically admissible set of displacements and $u_{0}$ is the prescribed displacement on the admissible Dirichlet boundary $\Gamma_{D}$ while $H(\bullet)$ is the Heaviside function, whose derived function $\delta(\bullet)$ is the Dirac delta function, $f(u, v)$ presents the strain energy density, which is written as $f(u, v)=\varepsilon_{i j}^{T}(u) E_{i j k l} \varepsilon_{k l}(v) / 2$, where $\varepsilon$ is the strain filed.

Introducing the concept of material derivative, the design sensitivities for the objective function and constraint are obtained as follows:

$$
\begin{aligned}
& \frac{\partial J(u, \Phi)}{\partial \alpha_{i}(t)}=\int_{\Omega} \gamma(u, \Phi) \varphi_{i}(\mathbf{x}) \delta(\Phi) d \Omega \\
& \frac{\partial G(\Phi)}{\partial \alpha_{i}(t)}=\int_{\Omega} \varphi_{i}(\mathbf{x}) \delta(\Phi) d \Omega
\end{aligned}
$$

At the same time, the Lagrangian method is utilized to convert the original constrained optimization problem into an unconstrained problem, and finally, a heuristic scheme can be derived to update the design variable:

$$
\begin{aligned}
& \alpha_{i}^{k+1}=\left\{\begin{array}{l}
\min \left[(m+1) \alpha_{i}^{(k)}, \alpha_{i, \max }\right], \text { if } \min \left[(m+1) \alpha_{i}^{(k)}, \alpha_{i, \max }\right] \leq\left[B_{i}^{(k)}\right]^{\zeta} \alpha_{i}^{(k)} \\
{\left[B_{i}^{(k)}\right]^{\zeta} \alpha_{i}^{(k)}, \text { if }\left\{\begin{array}{l}
\max \left[(1-m) \alpha_{i}^{(k)}, \alpha_{i, \min }\right]<\left[B_{i}^{(k)}\right]^{\zeta} \alpha_{i}^{(k)} \\
{\left[B_{i}^{(k)}\right]^{\zeta} \alpha_{i}^{(k)}<\min \left[(m+1) \alpha_{i}^{(k)}, \alpha_{i, \min }\right]}
\end{array}\right.} \\
\max \left[(1-m) \alpha_{i}^{(k)}, \alpha_{i, \min }\right], \text { if }\left[B_{i}^{(k)}\right]^{\zeta} \alpha_{i}^{(k)} \leq \max \left[(1-m) \alpha_{i}^{(k)}, \alpha_{i, \min }\right]
\end{array}\right. \\
& B_{i}^{(k)}=\frac{\partial J(u, \Phi)}{\partial \alpha_{i}^{(k)}} / \max \left(\mu, \Lambda \frac{\partial G(\Phi)}{\partial \alpha_{i}^{(k)}}\right)
\end{aligned}
$$

where the damping factor $\zeta(0<\zeta<1)$ as well as the moving limit $m(0<m<1)$ are the artificial parameters to stabilize the iterative process.

\section{Introduction on ANSYS secondary development}

There are four types of secondary tools for ANSYS, namely ANSYS Parameter Design Language (APDL), User Programmable Features (UPFs), User Interface Design Language (UIDL) and Tool Command Language (TCK/TK). In this paper, APDL and UPFs are used to develop structural topology optimization. A brief introduction about these two secondary development tools is given.

APDL is composed of general programming parts (parameters, arrays, vectors and matrix operations, branches, loops, repeats, etc.) that are similar to FORTRAN languages and more than 1000 ANSYS command streams. The most advantage of APDL is that it can easily achieve the repetitive work of model-building, grid-meshing, load-loading and post-processing, which can greatly reduce the workload when processing the topology optimization.

UPFs is the another ANSYS secondary development language which contains two parts: one is the user subroutine that can be modified by users, and the other is the general subroutine that the user 
cannot modify but can be called directly. In our work, we write the optimization algorithm of the PLSM through the user subroutine while calling on the ANSYS database though the general user subroutine.

The design of continuum structure topology optimization generally contains the follow four steps: a. initialization of structure design domain; b. finite element analysis; c. sensitivity analysis of optimization problems; $d$. update of design variable. In this paper, we apply the PLSM to the ANSYS topology optimization module not only take advantage of the powerful finite element analysis of ANSYS, but also gain clear structural boundary through PLSM. According to the above introduce of the APDL and UPFs, and the four steps of structure topology optimization designed as follow flowchart.

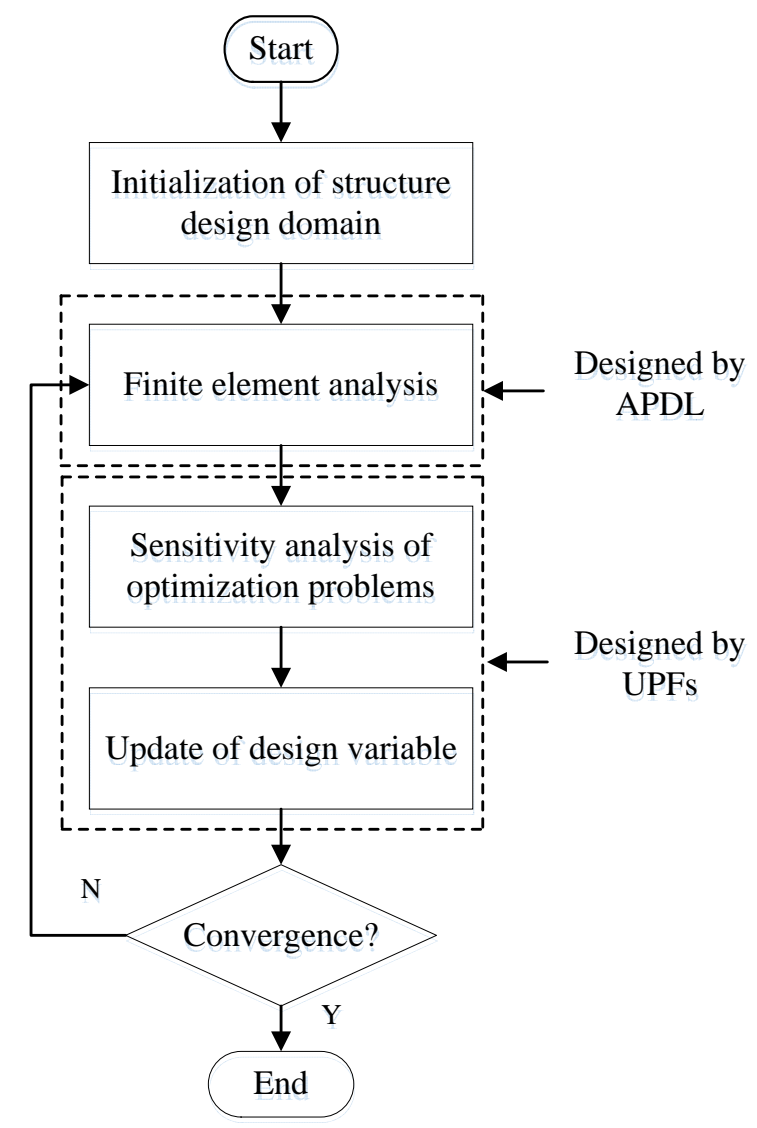

Fig. 2 Flowchart of topology optimization based on PLSM under ANSYS secondary development From the Fig. 2, it is clearly to see that the finite element analysis is designed by ADPL because it is a repeat work in the process of topology optimization, so using APDL can greatly reduce the workload of topology optimization. The last two steps are designed by UPFs, as we need to apply the algorithms based on PLSM to the ANSYS topology optimization module.

It should be pointed out that after writing the structural optimization programs of the PLSM using UPFs language, ANSYS software should be recompiled so that the user specific programs can be used in the ANSYS topology optimization module.

\section{Numerical examples}

To verity its feasibility and correctness, the adopted structural topology optimization approach based on PLSM under the environment of ANSYS secondary development is applied to optimize typical 2D and 3D examples. In the area of structural topology optimization, the optimal design of structural stiffness under single workload condition is considered to be the most typical topological optimization problem, so both of these examples are the maximum stiffness design problems. 2D and 3D examples are shown in the following sections. 
In the examples, the elastic module is equal to 180Gpa, and the Poisson's ratio is equal to 0.3 . The optimization process will be terminated when the convergence tolerances are less than 1e-4 and 5e-3 in the two-dimensional and three-dimensional optimization problems, respectively.

\subsection{Two-dimensional cantilever beam}

The design space of the two-dimensional cantilever beam is shown in Fig. 3 in which the ratio between length and width is 2 . The left side of the cantilever is fixed as the Dirichlet boundary and a concentrated loading which is equal to $10 \mathrm{KN}$ is considered in the center of the right side. The object is to minimize the structural strain energy (namely maximum structural stiffness) while the material usage is $50 \%$ in volume fraction.

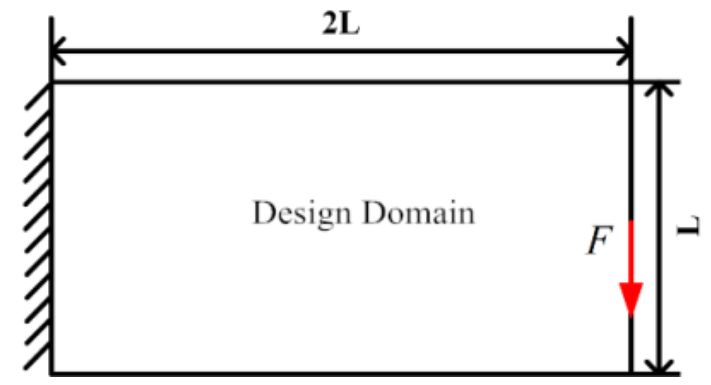

Fig. 3 Design domain of a two-dimensional cantilever beam

The structure shape of the initial design is shown in Fig. 4(a). Several regular holes are added into the entire design domain to speed up the iterative process. Fig. 4(b)-4(e) are the intermediate designs in the different iterations. The optimal result is shown in the Fig. 4(f). Figure 5 shows the optimization process in MATLAB as the same cantilever beam. It is obviously to see that the optimal results of cantilever beam between ANSYS and MATLAB are consistent. It is demonstrated that the produced structural topology optimization method based PLSM under the environment of ANSYS secondary development is reasonable and feasible.

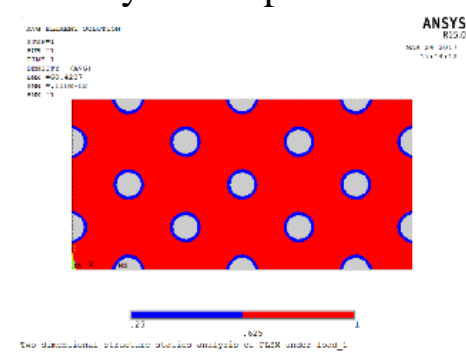

(a)

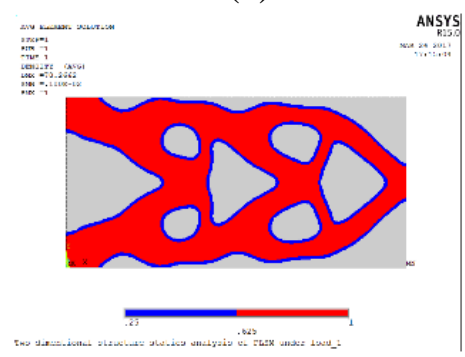

(d)

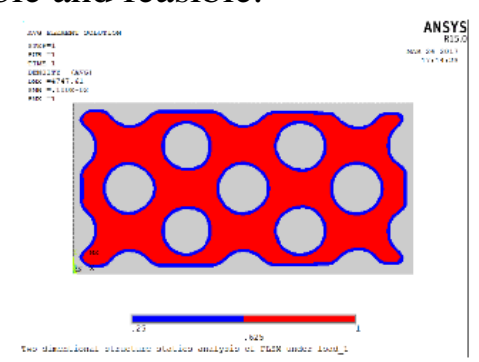

(b)

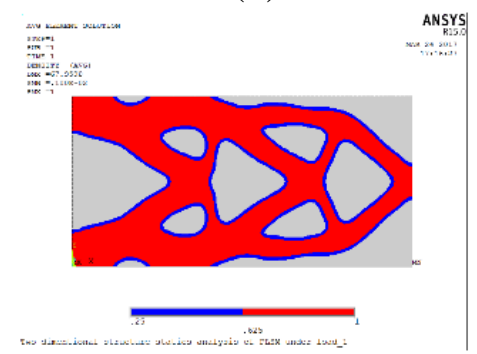

(e)

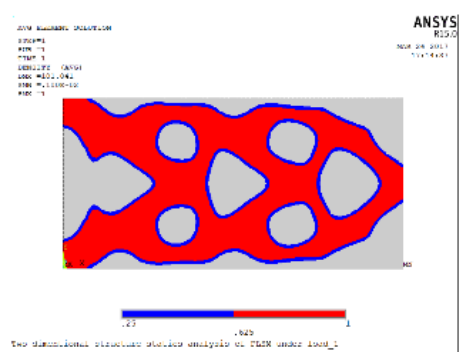

(c)

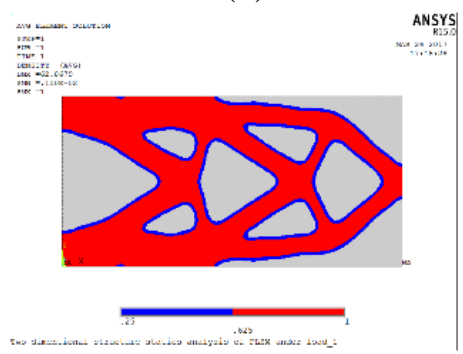

(f)

Fig. 4 Structural boundaries of a two-dimensional cantilever beam at different iterations: (a) initial design; (b) the 10th iteration; (c) the 20th iteration; (d) the 40th iteration; (e) the 100th iteration; (f) the final design 


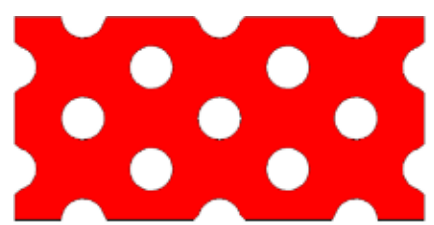

(a) initial design

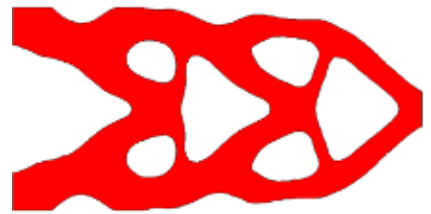

(d) $40^{\text {th }}$ iteration

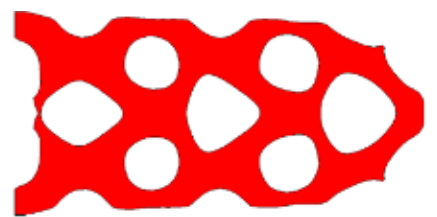

(b) $10^{\text {th }}$ iteration

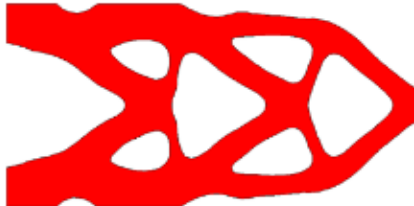

(e) $100^{\text {th }}$ iteration

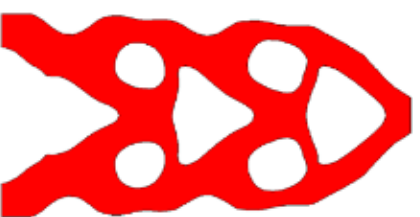

(c) $20^{\text {th }}$ iteration

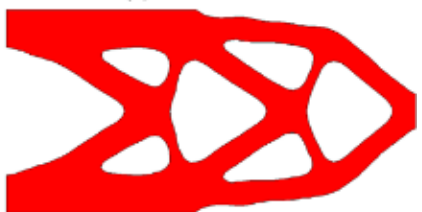

(f) finial design

Fig. 5 Structural boundaries at different iterations in MATLAB

Fig. 6(a) and 6(b) show the convergent history of objective function and the volume constraint respectively. It can be seen that the objective value increase during the first 10 iterations, because of the violation of volume constraint. After that, the volume fraction is pulled back to 0.5 , and the objective function starts to decrease stably. The optimal topology of the structure is achieved after 18 steps, and the following iterations are used to perform the shape optimization of structural boundary. It takes 187 steps to achieve the optimal strain energy value of 33.7253, which is the same as the numerical results in MATLAB. It further proves the correctness of the method produced in this paper.

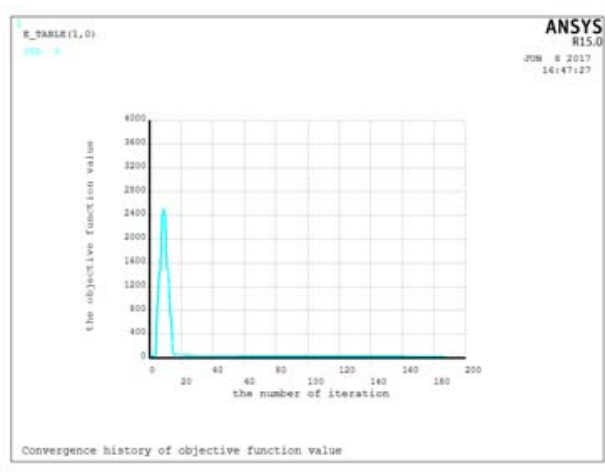

(a)

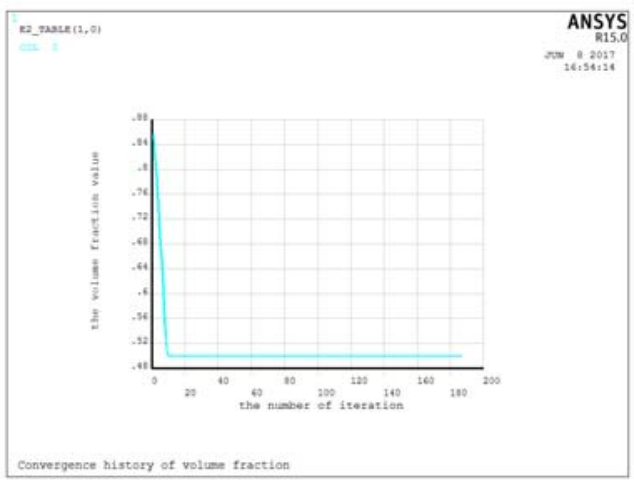

(b)

Fig. 6 Convergent histories of the cantilever beam: (a) objective function; (b) volume fraction

Above, we demonstrated the method is correct and reasonable though the optimal results of a cantilever beam both in ANSYS and MATLAB. The application of this method in 3D structure optimization is illustrated by two three-dimensional examples.

\subsection{Three-dimensional cantilever beam}

The design space of the three-dimensional cantilever beam 1 is shown in Fig. 7(a) in which the size of length, width and height are 2.0 meters, 0.4 meters and 1.0 meters, respectively. The left side of cantilever is fixed and a concentrated loading which equals to $300 \mathrm{KN}$ is considered in the center of the right side. Because of the symmetry, the width of model can be halved. The object is to minimize the structural strain energy (namely maximum structural stiffness) while the material usage is $50 \%$ in volume fraction.

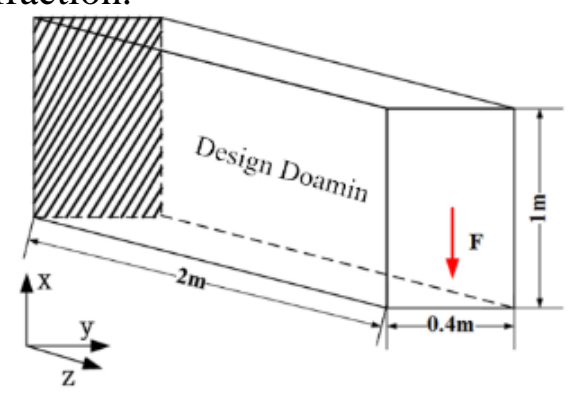

(a)

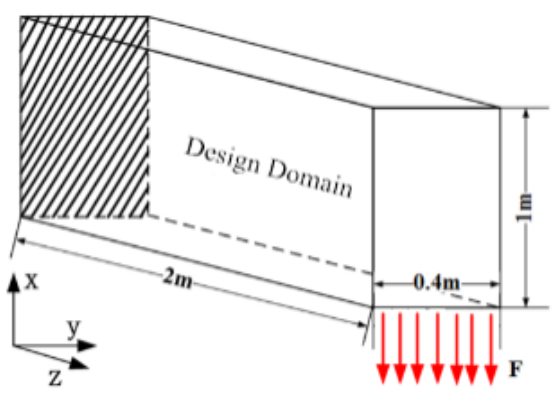

(b)

Fig. 7 Design domain of a three-dimensional structure: (a) a cantilever beam 1; (b) a cantilever beam 
The topology optimization results obtained by the ANSYS secondary development are shown in the Fig. 8. Figure 8(a) is the initial design of three-dimensional cantilever beam. Several regular holes were added into the entire design domain to speed up the iterative process. The optimal design result is shown in Fig. 8(f).

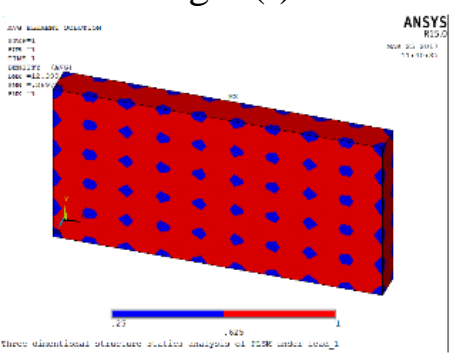

(a)

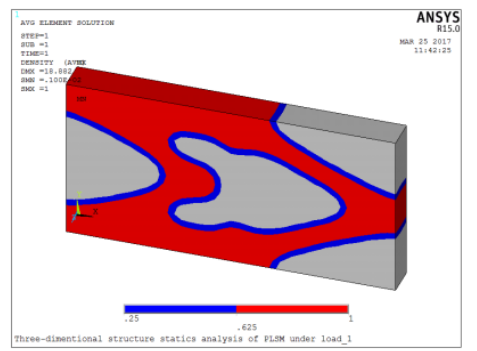

(d)

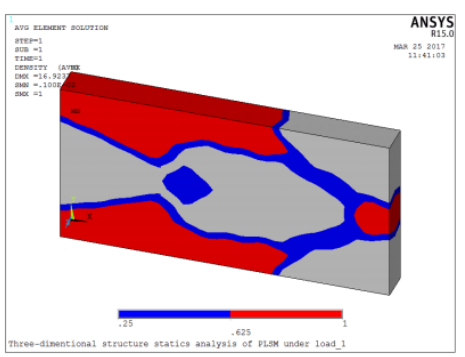

(b)

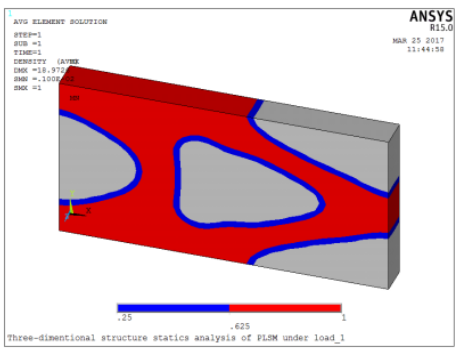

(e)

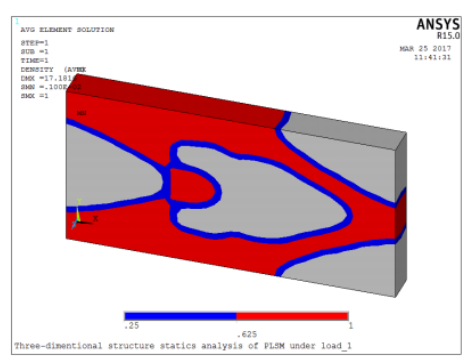

(c)

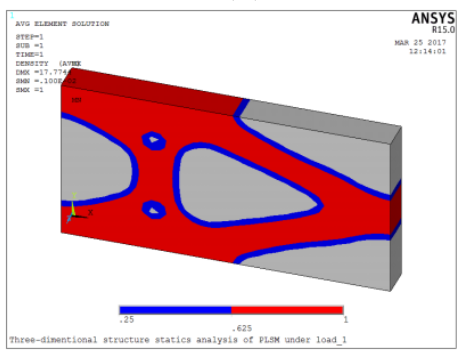

(f)

Fig. 8 Structural boundaries of a three-dimensional cantilever beam 1 at different iterations: (a) initial design; (b)-(e) intermediate design; (f) optimal design

The next example is the three-dimensional cantilever beam 2, the design space is the same as the cantilever beam 1, as shown in Fig. 7(b), and the only difference is that the load is a uniform loading rather than a concentrated loading and is considered in the bottom of the right end.

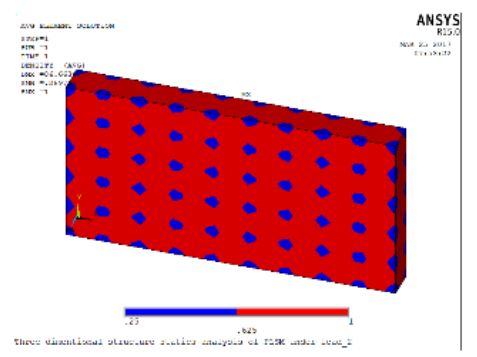

(a)

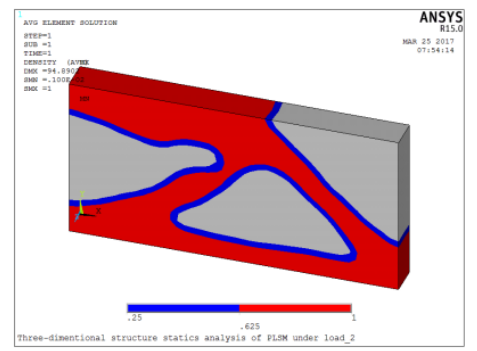

(d)

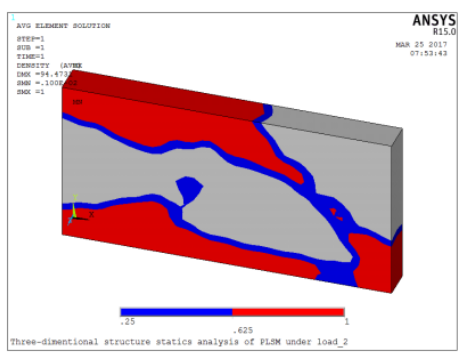

(b)

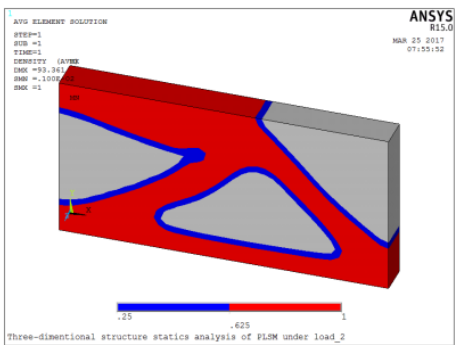

(e)

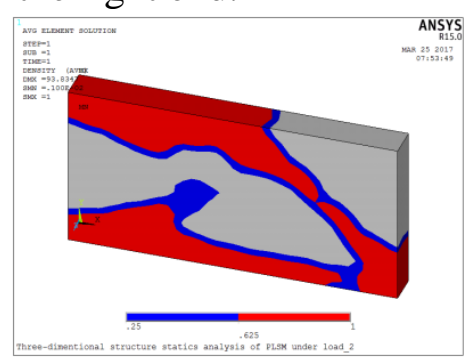

(c)

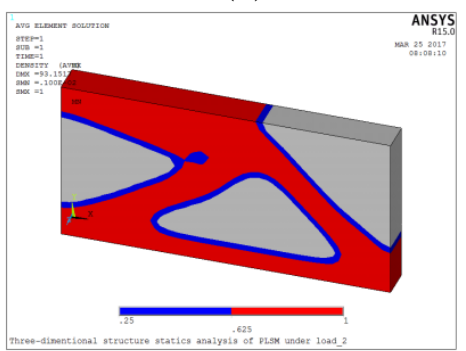

(f)

Fig. 9 Structural boundaries of a three-dimensional cantilever beam 2 at different iterations, (a) initial design; (b)-(e) intermediate design; (f) optimal design

The topology optimization results of three-dimensional cantilever beam 2 are shown in the Fig. 9. The initial design and the intermediate design are seen in Fig. 9(a)-9(e), respectively. The optimal design is shown in Fig. 9(f). 


\section{Conclusion}

In this paper, we realized the structural topology optimization based on PLSM under the environment of ANSYS secondary development. Through combining with the advantage of the explicit boundary of the PLSM and the strong finite element analysis capability of ANSYS, we expanded the practical applications of structural topology optimization. Finally, several typical 2D and 3D examples verified the correctness and feasibility of this method. This work can provide an important guidance for the ANSYS secondary development to realize the structural topology optimization.

\section{Acknowledgements}

This research was supported by the National Natural Science Foundation of China under grant Nos. 51675196 and 51421062, and the National Basic Scientific Research Program of China under grant No. JCKY2016110C012.

\section{References}

[1] Rozvany G.I.N. A critical review of established methods of structural topology optimization. Structural and Multidisciplinary Optimization. 2008, 37(3): 217-237.

[2] Bendsoe M.P., Sigmund O. topology Optimization: Theory, Methods, and Applications. Springer: Berlin, Heidelberg. 2003.

[3] Bendsoe M.P., Kikuchi N. Generating optimal topology in structural design using a homogenization method. Computer Methods in applied Mechanics and Engineering. 1998, 71: 197-224.

[4] Rozvany G.I.N., Kirsch U., Bendsoe M.P., et al. Layout optimization of structures. Applied Mechanics Reviews. 1995, 48(2): 41-119.

[5] Sigmund O. A 99 line topology optimization code written in Matlab. Structural and Multidisciplinary Optimization. 2001, 21(2): 120-127.

[6] Xie Y.M., Steven G.P. A simple evolutionary for structural optimization. Computer \& Structures. 1993, 49(5): 885-896.

[7] Sethian J.A., Wiegmann A. Structural boundary design via level set and immersed interface methods. Journal of Computational Physics. 2000, 163(2): 489-528.

[8] Wang M.Y., Wang X.M., Guo D.M. A level set method for structural topology optimization. Computer methods in Applied Mechanics and Engineering. 2003, 192: 227-246.

[9] Sigmund O., Petersson J. Numerical instabilities in topology optimization: A survey on procedures dealing with checkerboards, mesh-dependencies and local minima. Structural Optimization. 1998, 16(1): 68-75.

[10] Van Dijk N.P., Maute K., Langelaar M., et al. Level-set methods for structural topology optimization: a review. Structural and Multidisciplinary Optimization. 2013, 48(3): 437-472.

[11] Belytschko T., Xiao S.P., Parimi C. Topology optimization with implicity function and regularization. International Journal for Numerical Methods in Engineering. 2003, 57(8): 1177-1196.

[12] Luo J.Z., Luo Z., Tong L.Y., et al. A semi-implicit level set method for structural shape and topology optimization. Journal of Computational Physics. 2008, 227(11): 5561-5581.

[13] Buhmann M.D. Radial Basis Functions: Theory and Implementations, Cambridge Monographs on Applied and Computational Mathematics. vol. 12, Cambridge: Cambridge University Press. 2004.

[14] Wendland H. Computational Aspects of Radial Basis Function Approximation. Studies in Computational Mathematics. 2005, 12(12): 231-256.

[15] Junzhao L., Zhen L., Liping C., et al. A semi-implicit level set method for structural shape and topology optimization. Journal of Computational Physics. 2008, 227(11): 5561-5581. 
[16] Luo Z., Tong L., Wang M. Y., et al. Shape and topology optimization of compliant mechanisms using a parameterization level set method. Journal of Computational Physics. 2007, 227(1): 680-705.

[17] Sethian J.A. Level Set Methods and Fast Marching Methods: Evolving Interfaces in Computational Geometry. Fluid Mechanics, Computer Version and Material Science. Cambridge: Cambridge University Press. 1999.

[18] Luo Z., Wang M.Y., Wang S., Wei P. A level set-based parameterization method for structural shape and topology optimization. International Journal for Numerical methods in Engineering. 2008, 76(1): 1-26.

[19] Luo Z., Tong L.Y., Kang Z. A level set method for structural shape and topology optimization using radial basis functions. Computers \& Structures. 2009, 87(7-8): 425-434.

[20] Wang S., Wang M.Y. Radial basis functions and level set method for structural topology optimization. International Journal for Numerical Methods in Engineering. 2006, 65(12): 2060-2090.

[21] Wendland H. Piecewise polynomial, positive define and compactly supported radial functions of minimal degree. Advances in Computational Mathematics. 1995, 4(1): 389-396. 